\title{
A characterization of the Einstein tensor in terms of spinors
}

\author{
lan M. Anderson \\ Department of Mathematics. The University of Arizona, Tucson, Arizona 85721
}

David Lovelock

Department of Mathematics. The University of Arizona, Tucson, Arizona 85721 and Department of Applied Mathematics, University of Waterloo, Waterloo, Ontario, Canada N2L 3GI (Received 30 December 1975)

\begin{abstract}
All tensors of contravariant rank two which are divergence-free on one index, concomitants of a spinor field $\sigma_{i A X}$ together with its first two partial derivatives, and scalars under spin transformations are constructed. The Einstein and metric tensors are the only candidates.
\end{abstract}

\section{INTRODUCTION}

It is usually assumed that the field equations governing the interaction of the gravitational field with any other field (the latter having associated with it an energy-momentum tensor $T^{i j}$ ) are of the form

$$
A^{i j}=T^{i j},
$$

where $A^{i j}$ are the components of a type $(2,0)$ tensor which is constructed only from those field variables characterizing the gravitational field. Furthermore, it is customary to demand that the choice of $A^{i j}$ be restricted by the identity

$$
A^{i j}{ }_{1 j}=0 \text {, }
$$

in order that, as a consequence of (1.1), we have

$$
T^{i j}{ }_{1 j}=0 \text {. }
$$

Typically, if it is assumed that

(i) the gravitational field is characterized by a symmetric metric tensor $g_{a b}$, and

(ii) the quantities $A^{i j}$ are constructed from $g_{a b}$ and its first two partial derivatives, i.e.,

$$
A^{i j}=A^{i j}\left(g_{a b} ; g_{a b, c} ; g_{a b, c d}\right) \text {, }
$$

then it is known that, ${ }^{1}$ in a four-dimensional space, (1.2) and (1.3) imply that

$$
A^{i j}=a G^{i j}+b g^{i j},
$$

where $a, b$ are constants, and $G^{i j}$ is the Einstein tensor. In this case (1.4) and (1.1) give rise to the usual Einstein field equations, where $T^{i j}$ now satisfy

$$
T^{i j}=T^{i l} \text {. }
$$

However, it has been claimed by some, ${ }^{2,3}$ that $T^{i j}$ should be nonsymmetric, in which case, as has been pointed out by Ehlers, ${ }^{4}$ in order to accommodate this possibility, the assumptions (i) and (ii) would have to be changed. The purpose of this note is to discuss the consequence of adopting one possible alternative to (i) and (ii).

It is known that the gravitational field can be characterized in terms of a spinor field $\sigma_{i A X^{\prime}}$ or a tetrad field $h \quad(\alpha=1, \ldots, 4)$, these two characterizations being equivalent by virtue of the relations

$$
\sigma_{i A X^{\prime}}=\underset{(\alpha)^{i}}{h} \sigma_{\alpha A X^{\prime}}
$$

the $\sigma_{\alpha A X^{\prime}}$ being the conventional Pauli spin matrices. These quantities are related to the metric by

$$
g_{i j}=\sigma_{i A X^{\prime}} \sigma_{j B Y^{\prime}} \epsilon^{A B} \epsilon^{X^{\prime} Y^{\prime}}=\sigma_{i A X^{\prime}}, \sigma_{j}^{A X^{\prime}} .
$$

Guided by these observations, and (1.3), we shall therefore seek all tensors $A^{i j}$ satisfying (1.2) and for which

$$
A^{i j}=A^{i j}\left(\sigma_{a A X^{\prime}} ; \sigma_{a A X^{\prime}, b} ; \sigma_{a A X^{\prime}, b c}\right),
$$

where $A^{i j}$ are also assumed to be invariant under arbitrary (unimodular) spin transformations.

This problem is equivalent to finding all tensors $A^{i j}$ satisfying (1.2) and

$$
A^{i j}=A^{i j}\left(\underset{(\alpha)^{a}}{h} ; \underset{(\alpha)^{a, b}}{h} ; \underset{(\alpha)^{a, b c}}{h}\right),
$$

where $A^{i j}$ are scalars under arbitrary proper Lorentz transformations. Skew-symmetric tensors satisfying (1.2) and (1.8) are known: $:^{5}$ however they are not scalars under arbitrary proper Lorentz transformations,

It is clear from (1.6) that every spin-tensor which is a concomitant of $g_{i j}$ and its partial derivatives is always a concomitant of $\sigma_{i A X^{\prime}}$ and its partial derivatives. If the converse to this were valid, then the above problem, viz. (1.2) and (1.7) would immediately reduce to (1.2) and (1.3), and so, without further calculation (1.4) would follow. Unfortunately a proof of the converse does not seem to exist in the literature.

The spinor notation which we adopt here is essentially that of Pirani. ${ }^{6}$ From (1.6) it can be shown that the following is an identity (Schmutzer ${ }^{7}$ ):

$$
\begin{aligned}
\sigma^{a A Y^{\prime}} \sigma_{B Y^{\prime}} \sigma^{\infty B X^{\prime}} & =\frac{1}{2}\left(g^{a b} \sigma^{a A X^{\prime}}+g^{b c} \sigma^{a A X^{\prime}}\right. \\
& \left.-g^{a c} \sigma^{b A X^{\prime}}+i \epsilon^{a b c d} \sigma_{d} A X^{\prime}\right) .
\end{aligned}
$$

If we define $S^{a b}{ }_{A}^{B}$ and $S^{a b}{ }_{X^{\prime}} Y^{\prime}$ by

$$
\begin{aligned}
& S^{a b}{ }_{A}^{B}=\sigma_{A X^{\prime}}^{a} \sigma^{b B X^{\prime}}-\sigma_{A X^{\prime}}^{b} \sigma^{a B X^{\prime}}, \\
& S^{a b}{ }_{X^{\prime}}{ }^{*}=\sigma_{A X^{\prime}}^{a} \sigma^{b A Y^{\prime}}-\sigma_{A X^{\prime}} \sigma^{a A Y^{*}},
\end{aligned}
$$

then repeated application of $(1.9)$ gives $r$ ise to

Copyright $\oplus 1976$ American Institute of Physics 


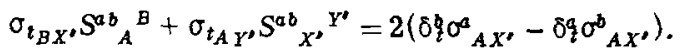

\section{CHARACTERIZATION OF $A^{\text {rs }}$}

Because the $A^{i f}$ are assumed to satisfy (1.7) and to be the components of a type $(2,0)$ tensor under arbitrary coordinate transformations, certain invariance identities ${ }^{8}$ must be satisfied, one of which is

$$
A^{r s ; a A X^{\prime}, b c}+A^{r s i c A X^{\prime}, a b}+A^{r s ; b A X^{\prime}, c a}=0,
$$

where $A^{r s i a A X^{\prime}, b c}=\partial A^{r s} / \partial \sigma_{a A X^{\prime}, b c}$ is a spin-tensor symmetric in $b c$.

The invariance of $A^{i j}$ under spinor transformations also gives rise to invariance identities, ${ }^{8}$ two of which are

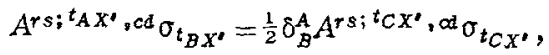

and

$$
A^{r s ; t_{A X^{\prime}, c d}} \sigma_{t_{A} Y^{\prime}}=\frac{1}{2} \delta_{Y^{\prime}}^{X^{\prime} A^{r s ;} t_{A} Z^{\prime}, c d} \sigma_{t_{A Z^{\prime}}}
$$

If we define the spin-tensor $A^{r s ; a b, c d}$ by

$$
A^{r s ; a b, c d}=A^{r s ; a A X^{\prime}, c d} \sigma_{A X^{*}}^{b},
$$

then (2.1) gives rise to

$$
A^{r s ; a b, c d}+A^{r s ; d b, a c}+A^{r s ; c b, d a}=0 .
$$

If we multiply $(2.2)$ by $S^{a b}{ }_{A}^{B},(2.3)$ by $S^{a b}{ }_{X^{*}} y^{*}$ and add the resulting equations we find, by virtue of $(1.12)$,

$$
A^{r s ; a b, c d}=A^{r s i b a, c d} \text {. }
$$

From (2.5) and (2.6) it is easily seen that

$$
A^{r s ; a b, c t}=A^{r s i c d, a b} \text {. }
$$

Up to the present, no use has been made of (1.2), which in view of $(1.7)$ can be expressed in the form

$A^{r s ; a A X^{\prime}, a d} \sigma_{a A X^{\prime}, a d s}+O^{r}\left(\sigma_{a A X^{\prime}} ; \sigma_{a A X^{\prime}, b} ; \sigma_{a A X^{\prime}, b c}\right)=0$.

Differentiation of (2.8) with respect to $\sigma_{a A X^{*} \text {, ss }}$ thus yields

$$
A^{r s ; a A X^{\prime}, c d}+A^{r d ; a A X^{\prime}, s c}+A^{r c ; a A X^{\prime}, d s}=0,
$$

which, by (2.4), is equivalent to

$$
A^{r s ; a b, \alpha d}+A^{r d ; a b, s c}+A^{r c ; a b, d s}=0 .
$$

If we define $A^{r s ; a b, c d ; 1 j, k t}$ by

$$
A^{r s ; a b, c d ; i j, k l}=\left(\partial A^{r s ; a b, c d} / \partial \sigma_{i B Y^{*}, k l}\right) \sigma_{B Y^{*}},
$$

and note (2.4), we see that

$$
A^{r s ; a b, c d i t j, k l}=A^{r s ; i j, k l ; a b, c d} \text {. }
$$

Elsewhere ${ }^{l}$ it has been shown that, in a four-dimensional space, if $A^{r s ; a b, c d ; i, k t}$ is any quantity which has the properties $(2.5),(2.6),(2.7),(2.9)$, and (2.11) then

$$
A^{r s i a b, c d ; i f, k t}=0 \text {. }
$$

[An alternative proof of (2.12), which readily suggests generalizations to higher dimensional spaces, is presented in the Appendix.]

A comparison of (2.12) and (2.10) establishes that $A^{i j ; a b, c d}$ is independent of $\sigma_{i B Y^{\circ}, k l}$, which, in turn, implies that $A^{t f ; a b, c d}$ is also independent of $\sigma_{i B Y^{\prime}, k},{ }^{9}$ i.e.,

$$
A^{r s i a b, c d}=B^{r s a b c d}\left(\sigma_{t A X^{\prime}}\right),
$$

where $B^{\text {rsabcd }}$ is a spin-tensor with the same symmetry properties as $A^{r s ; a b, c d}$, viz. (2.5), (2.6), (2.7), and (2.9).

From (2.4) and (2.13) we thus find

$$
A^{r s ; a A X^{\prime}, \propto d}=B^{r a b c d} \sigma_{b}^{A X^{\prime}} \text {, }
$$

which, upon integration, yields

$A^{r s}=B^{r s a b d} \sigma_{b}{ }^{A X^{\prime}} \sigma_{a A X^{\prime}, c d}+C^{r s}\left(\sigma_{a A X^{\prime}} ; \sigma_{a A X^{\prime}, b}\right)$.

From (1.6) we see that

$g_{a b, c d}=\sigma_{a A X^{\prime}, c d} \sigma_{b}^{A X^{\prime}}+\sigma_{a}{ }^{A X^{\prime}} \sigma_{b A X^{\prime}, c d}+O_{a b c d}\left(\sigma_{i A X^{\prime}} ; \sigma_{i A X^{\prime}, j}\right)$.

When this is taken together with (2.14), it gives rise to

$$
A^{r s}=\frac{1}{2} B^{r s a b c d} g_{a b, c d}+D^{r s}\left(\sigma_{a A X^{\prime}} ; \sigma_{a A X^{\prime}, b}\right),
$$

which can be reexpressed ${ }^{10}$ in the form

$$
A^{r s}=\frac{1}{3} B^{r s a b c d} R_{c a b d}+B^{r s}\left(\sigma_{a A X^{\prime}} ; \sigma_{a A X^{\prime}, b}\right)
$$

where $B^{r s}$ is now a spin-tensor, which is therefore independent of $\sigma_{a A X^{\prime}, b}$, i,e.,

$$
A^{r s}=\frac{1}{3} B^{r s a b c d} R_{c a b d}+B^{r s}\left(\sigma_{a A X}\right) .
$$

All spin-tensors $B^{r s}=B^{r s}\left(\sigma_{a A X^{e}}\right)$ have been constructed ${ }^{9}$ the result being

$$
B^{r s}=b g^{r s} \text {, }
$$

where $b$ is a real constant.

Consequently, the problem of determining $A^{r s}$ has reduced to the evaluation of the spin-tensor $B^{\text {sabcd }}$ where $B^{r s a b c d}=B^{r s a b c a}\left(\sigma_{i A X^{\prime}}\right)$ and where $B^{r s a b c d}$ satisfies $(2,5)$, (2.6), (2.7), and (2.9). To do this we proceed somewhat indirectly, as follows.

It is easily seen that, because of the symmetry properties of $B^{r a b c d}$,

$$
B^{r t_{a}\{c j} \delta_{t_{i j}^{b d}}^{s b}=2\left(B^{r s a b c d}+B^{r b a d c s}+B^{r d a s c b}\right),
$$

from which we obtain

$B^{r t_{a i c f}} \delta_{t i j}^{s b d}+B^{r t b i c f} \delta_{t i j}^{s a d}+B^{r t a\{d j} \delta_{t i j}^{s b c}+B^{r t b i d j} \delta_{t_{i j} j}^{s a c}=12 B^{r s a b c d}$.

If we define

$$
\beta_{i}^{a}{ }^{c}=-\frac{1}{\Gamma 2} \epsilon_{t i j i} B^{r t a i c j}
$$

and recall that

$$
\delta_{t i j}^{s b d}=-\epsilon^{s b d} \epsilon_{t i j l},
$$

we see that (2.17) reads

$B^{r s a b c d}=\epsilon^{s b d l} \beta^{r a}{ }_{l}{ }^{c}+\epsilon^{s a d l} \beta^{r b}{ }_{1} c+\epsilon^{s b c t} \beta^{r a d}{ }_{l}^{d}+\epsilon^{s a c t} \beta^{r b}{ }_{l}^{d}$.

Consequently a knowledge of $\beta^{a}{ }_{i}^{c}$ determines $B^{r a b c d}$.

From (2.18) we note that

$$
\beta^{r a}{ }_{i}^{c}=\beta^{r a}{ }_{i}^{c}\left(\sigma_{i A X^{\prime}}\right)
$$

and

$$
\begin{aligned}
& \beta^{r a}{ }_{i}{ }^{c}=-\beta^{r c}{ }_{l}^{a} \\
& \beta^{r a}{ }_{l}=0 .
\end{aligned}
$$

We now define 


$$
\beta_{A B C D X^{\prime} Y^{\prime} Z^{\prime} W^{\prime}}=\sigma_{r A X^{\prime}}, \sigma_{a B X^{\prime}}, \sigma_{c C Z^{\prime}}, \sigma_{D W^{2}} \mathrm{~B}^{r a}{ }_{i}{ }^{c},
$$

in which case (2.21) and (2.22) imply

$$
\beta_{A B C D X^{\prime} Y^{\prime} Z^{\prime} W^{\circ}}=-\beta_{A C B D X^{\prime} Z^{\prime} Y^{*} W^{*}},
$$

and

$$
\epsilon^{B D} \epsilon^{Y^{\prime} W^{\prime}} B_{A B C D X^{\prime} Y^{\prime} Z^{\prime} W^{\prime}}=0,
$$

respectively. From arguments similar to those presented in Lemma A3 of Ref. 9, we find by virtue of $(2.24)$ and $(2.25)$

$$
\begin{aligned}
& \beta_{A B C D X^{\prime} Y^{\prime} Z^{\prime} W^{\prime}}=i c\left[\epsilon_{B C^{\prime}} \epsilon_{A D}\left(\epsilon_{X^{\prime} Y^{\prime}} \epsilon_{Z^{\prime} W^{\prime}}+\epsilon_{X^{\prime} Z^{\prime}} \epsilon_{Y^{\prime} W^{\prime}}\right)\right. \\
& \left.-\epsilon_{Y^{\prime} Z^{\prime}} \epsilon_{X^{\prime} W^{\prime}}\left(\epsilon_{A B} \epsilon_{C D}+\epsilon_{A C} \epsilon_{B D}\right)\right],
\end{aligned}
$$

where $c$ is a real constant. The latter is substituted in (2.23) which is then solved for $\beta^{r a}{ }_{i}^{c},(1.9)$ being used repeatedly in the process, this giving rise to

$$
\beta^{r a}{ }_{l}^{c}=\frac{1}{3} a \epsilon^{r a c m} g_{l m},
$$

where $a$ is a real constant. Equations (2.16), (2.19), and $(2.26)$ are now substituted in $(2.15)$ to yield

$$
A^{r s}=a G^{r s}+b g^{r s} \text {. }
$$

We thus have the following theorem.

Theorem: If $A^{r s}=A^{r s}\left(\sigma_{a A X^{\prime}} ; \sigma_{a A X^{*}, b} ; \sigma_{a A X^{\prime}, b c}\right)$ is a spintensor and

$$
A^{r r}{ }_{\text {is }}=0 \text {, }
$$

then

$$
A^{r s}=a G^{r s}+b g^{r s}
$$

where $a, b$ are constants.

Consequently, we see that, even in this case, the symmetry of $A^{r s}$ [and hence of $T^{r s}$ by (1.1)] is again an inevitable consequence.

\section{APPENDIX}

The purpose of this appendix is to outline an alternative derivation of Eq. (2.12) which explicitly exhibits the role played by the dimensionality of the space. We begin by introducing quantities $\alpha_{a b}$ and $\beta_{i f}$ which are both assumed to be symmetric in their indices but are otherwise arbitrary, and consider the expression

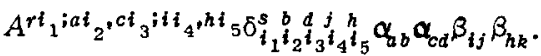

By expanding the Kronecker delta and by repeatedly invoking the symmetries of $A^{r s ; a b, c d ; i, h k}$, viz. (2.5), (2.6), (2.7), and (2.9), as well as the symmetries induced by the expression $\alpha_{a b} \alpha_{c d} \beta_{i j} \beta_{h k}$ a lengthy, but nevertheless straightforward calculation, shows that

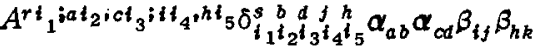

$$
\begin{aligned}
& =\frac{9}{2}\left[4 A^{r s ; a b, c d ; i j, h k}+A^{r s i a b_{,} i j ; c d, l k}\right] \alpha_{a b} \alpha_{c d} \beta_{t j} \beta_{h k} .
\end{aligned}
$$

This equation holds irrespective of the dimension of the underlying space. However, for $n=4$, the left-hand side of (A1) vanishes identically in which case it is easily seen, on account of the arbitrary nature of the $\alpha_{a b}$ and $\beta_{i j}$, that

$8 A^{r s ; a b, c d ; i j, h k}+A^{r s i a b, i j ; h k, c d}+A^{r s ; a b, h k ; c d, t j}=0$.

By successively interchanging the pairs $(c d),(i j)$, and $(h k)$ in (A2), it thus follows that

$$
A^{r s ; a b, c d ; i j, h k}=0
$$

as required.

\section{ACKNOWLEDGMENTS}

This work was supported in part by a grant from the National Research Council of Canada. One of us (I. A.) would also like to thank the National Research Council for the award of a postgraduate scholar ship.

${ }^{1}$ D. Lovelock, J. Math. Phys. 13, 874 (1972).

${ }^{2} \mathrm{O}$. Costa de Beauregard, "Translational Inertial Spin Effect," in Perspectives in Geometry and Relativity. Hlavaty Festschrift (University of Indiana Press, Bloomington, 1966), p. 44.

${ }^{3}$ D.W. Sciama, Proc. Cambridge Philos. Soc. 54, 72 (1958). ${ }^{4} \mathrm{~J}$. Ehlers, Survey of General Relativity Theory. Relativity, Astrophysics and Cosmology (Reidel, Holland, 1973), p. 1. ${ }^{5}$ B. O. J. Tupper, Can. Math. Bull. 16, 111 (1972).

${ }^{6}$ F. A. E. Pirani, in Lectures on General Relativity, Summer Institute in Theoretical Physics, Brandeis (Prentice-Hall, Englewood Cliffs, N.J., 1965), Vol. 1, p. 249.

E. Schmutzer, Relativistische Physik (Akademische Verlaggesellschaft, Leipzig, 1968).

${ }^{8} \mathrm{~J}$. Wainwright, Tensor 19, 217 (1968).

'T.M. Anderson, Gen. Rel. Grav. 6, 621 (1974).

${ }^{10} \mathrm{D}$. Lovelock and H. Rund, Tensors, Differential Forms and Variational Principles (Wiley-Interscience, New York, 1975). 